\title{
Current and noise suppression in ac-driven coherent transport
}

\author{
Sigmund Kohler*, Michael Strass*, Peter Hänggi*, Miguel Rey ${ }^{\dagger}$ and \\ Fernando Sols** \\ *Institut für Physik, Universität Augsburg, Universitätsstraße 1, D-86135 Augsburg, Germany \\ ${ }^{\dagger}$ Departamento de Física Teórica de la Materia Condensada, Universidad Autónoma de Madrid, \\ E-28049 Madrid, Spain \\ ** Departamento de Física de Materiales, Universidad Complutense de Madrid, E-28040, Spain
}

\begin{abstract}
.
We investigate the possibility to manipulate for the transport through heterostructures the dc current and its noise properties by an ac gate voltage. For a computation of the noise strength, we map the system to a tight-binding model for which noise suppression by ac fields has been predicted recently. The quality of this description is tested by comparing the transmission of the tight-binding system with a transfer-matrix approach.
\end{abstract}

Keywords: coherent transport, driven systems, noise

PACS: 05.60.Gg, 05.40.-a, 72.40.+w 73.63.-b,

\section{INTRODUCTION AND MODELING}

Semiconductor heterostructures represent a popular physical system for the investigation of mesoscopic transport and tunneling phenomena [1, 2]. In particular, these setups open various ways to study tunneling in time-dependent systems [3-7]. A straightforward possibility for introducing a time-dependence is the application of an ac transport voltage which only modulates the energies of the electrons in the leads while the potential inside the mesoscopic region remains time-independent. This kind of driving allows for a description within the Tien-Gordon theory [8] expressing the dc current in terms of the static transmission and an effective distribution function for the lead electrons. If the time-dependence enters via an external microwave field or an ac gate voltage, however, such an approach is generally insufficient [9]. A remarkable difference with respect to the static situation is the emergence of inelastic transport channels stemming from the emission or absorption of quanta of the driving field. This follows indeed from a recently presented Floquet theory for the transport through driven tight-binding systems [7, 9]. For the computation of the dc current, the latter approach justifies the applicability of a Landauer-like current formula where the static transmission is replaced by the timeaveraged transmission of the time-dependent system.

The transmission of the transport channels can depend sensitively on the driving parameters; the contribution of certain channels can even vanish. For the transport across two barriers which enclose an oscillating potential well, Wagner [10] showed that it is possible to suppress the contribution of individual inelastic scattering channels. The total current, however, is given by the sum over all channels, and thus it is not possible 


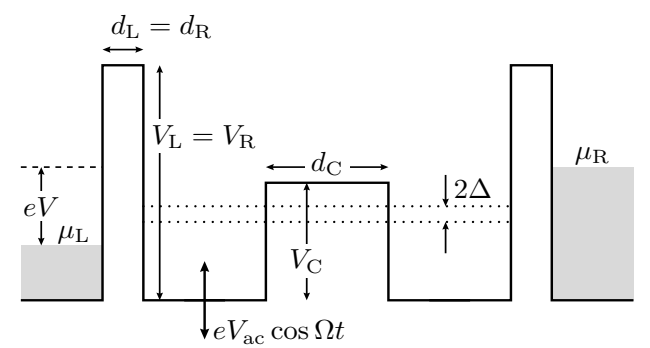

FIGURE 1. Model potential for the double-well heterostructure. In the numerical calculations, we employ barriers with the heights $V_{\mathrm{L}}=V_{\mathrm{R}}=90 \mathrm{meV}, V_{\mathrm{C}}=40 \mathrm{meV}$ and the widths $d_{\mathrm{L}}=d_{\mathrm{R}}=5 \mathrm{~nm}$, $d_{\mathrm{C}}=15 \mathrm{~nm}$. The dotted lines mark the energy of a metastable tunnel doublet with splitting energy $2 \Delta$ described by the Hamiltonian (2). The on-site energy of the left well is subject to an oscillating gate voltage with amplitude $V_{\text {ac }}$.

to isolate the contribution of a single channel in a current measurement. By contrast, in the case of transport through a two-level system with attached leads, driving with a dipole field has directly observable consequences: There, the driving not only affects the contribution of individual transport channels, but the dc current can be suppressed almost entirely $[11,12]$. For the appearance of this coherent current suppression, it is essential that the central region consists of at least two weakly coupled wells which oscillate relative to each other [9].

Here, we explore the possibility of coherent current suppression in double-well heterostructures. Thereby, we compare two theoretical approaches to describe coherent transport in quantum-well structures: The transfer-matrix method and a tight-binding approach. As a model we consider the triple-barrier structure sketched in Fig. 1 where the driving enters via an oscillating gate voltage which modulates the bottom of the left well. The applied transport voltage is assumed to shift the Fermi energy of the left lead by $-e V,-e$ being the electron charge. Having a tight-binding approximation for the double-well system at hand, we are able to compute within the recently developed Floquet approach $[7,13]$ also the noise strength.

\section{Transfer-matrix method}

Following Landauer [14], we consider the coherent mesoscopic transport as a quantum mechanical scattering process. The central idea of this approach is the assumption that sufficiently far from the scattering region, the electronic single-particle states are plane waves and that their occupation probability is given by the Fermi function with the chemical potential depending on the applied voltage. The unitarity of evolution under coherent ac driving allows us to write the resulting currents as [15]

$$
I=\frac{e}{h} \int \mathrm{d} E\left[T_{\mathrm{RL}}(E) f_{\mathrm{L}}(E)-T_{\mathrm{LR}}(E) f_{\mathrm{R}}(E)\right],
$$


where $T_{\mathrm{RL}}(E)$ denotes the total transmission probability of an electron with energy $E$ from the left to the right lead. The term $T_{\mathrm{LR}}(E)$ describes the respective scattering from the right to the left lead. These transmission probabilities comprise the sum over transverse modes and outgoing inelastic channels and can be computed with the transfer matrix method developed in Ref. [16]. The latter method relies on the fact that for a time-dependent spatially constant potential, the eigenfunctions are plane waves with an additional time-dependent phase factor. The propagator for a piecewise constant potential, in turn, can be constructed by imposing proper matching conditions at the boundaries of the adjacent layers of the heterostructure.

\section{Tight-binding approximation}

A different approach to study resonant tunneling in a driven double-well structure is based on the adoption of a tight-binding approximation where each well is represented by a single localized electron orbital. Then, the Hamiltonian of the transport setup reads $H(t)=H_{\text {wells }}(t)+H_{\text {leads }}+H_{\text {contacts }}$, where

$$
H_{\mathrm{wells}}(t)=-\Delta\left(c_{\mathrm{L}}^{\dagger} c_{\mathrm{R}}+c_{\mathrm{R}}^{\dagger} c_{\mathrm{L}}\right)+e V_{\mathrm{ac}} \cos (\Omega t) c_{\mathrm{L}}^{\dagger} c_{\mathrm{L}}
$$

describes the electrons in the wells. The second term of the Hamiltonian (2) accounts for the harmonic driving of the traversing electrons in the left well via an oscillating gate voltage with amplitude $V_{\text {ac }}$ and period $\mathscr{T}=2 \pi / \Omega$. The leads are modeled as ideal Fermi gases with the Hamiltonian $H_{\text {leads }}=\sum_{\ell, q} \varepsilon_{\ell q} c_{\ell q}^{\dagger} c_{\ell q}$, where $c_{\ell q}$ annihilates an electron in the lead with energy $\varepsilon_{\ell q}$ with $\ell=\mathrm{L}, \mathrm{R}$. As an initial condition, we employ the grand-canonical ensembles of electrons in the leads at inverse temperature $\beta=1 / k_{B} T$. Therefore, the lead electrons are characterized by the equilibrium Fermi distribution $f_{\ell}\left(\varepsilon_{\ell q}\right)=\left\{1+\exp \left[-\beta\left(\varepsilon_{\ell q}-\mu_{\ell}\right)\right]\right\}^{-1}$. The localized state in each well couples via the tunneling matrix element $V_{\ell q}$ to the state $|\ell q\rangle$ in the respective lead. The Hamiltonian which describes this interaction has the form $H_{\text {contacts }}=\sum_{\ell, q} V_{\ell q} c_{\ell q}^{\dagger} c_{\ell}+$ H.c. The leadwell coupling is entirely specified by the spectral density $\Gamma_{\ell}(\varepsilon)=2 \pi \sum_{q}\left|V_{\ell q}\right|^{2} \delta\left(\varepsilon-\varepsilon_{\ell q}\right)$. Since, for the system at hand, the bandwidth of the conduction band of the leads is much larger than the energy regime where transport happens, the spectral densities are practically constant, i.e. $\Gamma_{\ell}(\varepsilon)=\Gamma_{\ell}$, which defines the so-called wide-band limit. By matching for the static case the transmissions of the transfer-matrix and the tight-binding approach, we find the tight-binding parameters $\Gamma_{\mathrm{L}}=\Gamma_{\mathrm{R}}=0.16 \mathrm{meV}$ and $\Delta=0.23 \mathrm{meV}$.

\section{Floquet transport theory}

Starting from the Heisenberg equations of motion for the annihilation operators, one eliminates the lead operators and thereby obtains for the electrons on the dots a reduced set of equations. These are solved with the help of the retarded Green function obeying $[\mathrm{i} \hbar \mathrm{d} / \mathrm{d} t-\mathscr{H}(t)+\mathrm{i} \Gamma / 2] G\left(t, t^{\prime}\right)=\delta\left(t-t^{\prime}\right)$, where $\mathscr{H}(t)$ is the single-particle Hamiltonian corresponding to double well Hamiltonian (2) and $\Gamma=\Gamma_{L}=\Gamma_{R}$. 
The coefficients of the equation of motion for $G\left(t, t^{\prime}\right)$ are $2 \pi / \Omega$-periodic and, consequently, its solution can be constructed with the help of the Floquet ansatz $\left|\psi_{\alpha}(t)\right\rangle=$ $\exp \left[\left(-\mathrm{i} \varepsilon_{\alpha} / \hbar-\gamma_{\alpha}\right) t\right]\left|\phi_{\alpha}(t)\right\rangle$. The Floquet states $\left|\phi_{\alpha}(t)\right\rangle$ obey the time-periodicity of the Hamiltonian and fulfill the eigenvalue equation

$$
\left[\mathscr{H}(t)-\frac{\mathrm{i}}{2} \Gamma-\mathrm{i} \hbar \frac{\mathrm{d}}{\mathrm{d} t}\right]\left|\phi_{\alpha}(t)\right\rangle=\left(\varepsilon_{\alpha}-\mathrm{i} \hbar \gamma_{\alpha}\right)\left|\phi_{\alpha}(t)\right\rangle .
$$

This yields the retarded Green function $G\left(t, t^{\prime}\right)=-(\mathrm{i} / \hbar) \sum_{\alpha}\left|\psi_{\alpha}(t)\right\rangle\left\langle\psi_{\alpha}^{+}\left(t^{\prime}\right)\right| \Theta\left(t-t^{\prime}\right)$.

In particular, one finds for the current a convenient Landauer-like expression with an additional sum over the Fourier index $k$, i.e. $T_{\mathrm{LR}}(E)=\sum_{k} T_{\mathrm{LR}}^{(k)}(E)$ [7, 13]. Since the symmetrized noise correlation function $S\left(t, t^{\prime}\right)=\left\langle\left[I(t), I\left(t^{\prime}\right)\right]_{+}\right\rangle$depends explicitly on both times, we characterize the noise by the time-average of its zero-frequency component, $S=(\Omega / 2 \pi) \int_{0}^{2 \pi / \Omega} d t \int_{-\infty}^{+\infty} d \tau S(t, t-\tau)$.

\section{High-frequency approximation}

The Floquet treatment of the present transport problem allows for the implementation of a stationary perturbation scheme for driving frequencies much larger than all other frequency scales of the system [17]. This approach has recently been extended to transport situations which are characterized by the presence of leads [9, 12]; here we only mention the cornerstones of this approach and refer the reader to Ref. [7]. The starting point is the unitary transformation

$$
U_{0}(t)=\exp \left\{-\frac{\mathrm{i} e}{\hbar \Omega} V_{\mathrm{ac}} \sin (\Omega t) c_{\mathrm{L}}^{\dagger} c_{\mathrm{L}}\right\},
$$

which is first applied to the quantum-well Hamiltonian (2). For sufficiently large driving frequencies $\Omega \gg \Delta / \hbar$, a separation of time scales is performed. Thereby, fast oscillations of the transformed Hamiltonian are neglected by averaging over a driving period. Finally, we arrive at the effective Hamiltonian for the quantum wells

$$
\bar{H}_{\text {eff }}=\frac{1}{\mathscr{T}} \int_{0}^{\mathscr{T}} \mathrm{d} t\left(U_{0}^{\dagger} H_{\text {wells }}(t) U_{0}-\mathrm{i} \hbar U_{0}^{\dagger} \dot{U}_{0}\right)=-\Delta_{\text {eff }}\left(c_{\mathrm{L}}^{\dagger} c_{\mathrm{R}}+c_{\mathrm{R}}^{\dagger} c_{\mathrm{L}}\right)
$$

which is of the same form as in the static case but with the effective tunneling matrix element $\Delta_{\text {eff }}=J_{0}\left(e V_{\mathrm{ac}} / \hbar \Omega\right) \Delta, J_{0}$ being the zeroth order Bessel function of the first kind.

The transformation (4) also affects the lead-well coupling. Applying $U_{0}(t)$ to $H_{\text {contacts }}$ and solving the Heisenberg equations for the lead and quantum-well operators in the wide-band limit, we find that the influence of the left lead is no longer determined by the Fermi function $f_{\mathrm{L}}(\varepsilon)$ but rather by the effective electron distribution [7, 13]

$$
f_{\mathrm{L}, \mathrm{eff}}(\varepsilon)=\sum_{n=-\infty}^{\infty} J_{n}^{2}\left(\frac{e V_{\mathrm{ac}}}{\hbar \Omega}\right) f_{\mathrm{L}}(\varepsilon+n \hbar \Omega) .
$$

The squares of the $n$ th-order Bessel function of the first kind $J_{n}$ weight the processes where an electron with energy $\varepsilon$ is transmitted under the emission $(n<0)$ or absorption $(n>0)$ of $|n|$ photons. 


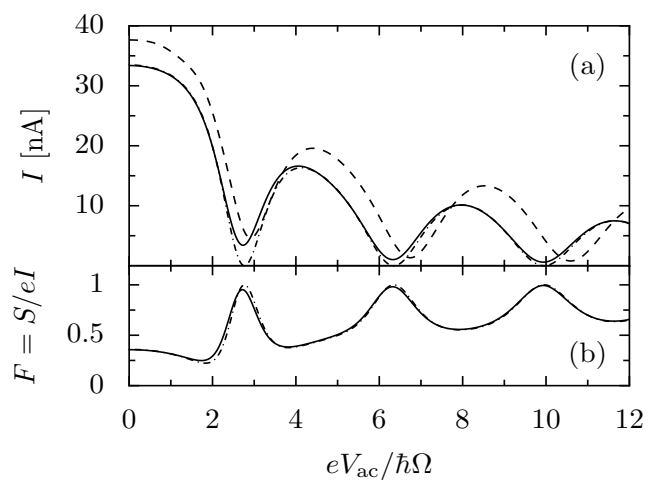

FIGURE 2. (a) Average current $v s$. driving amplitude obtained numerically from tight-binding (solid line) and transfer-matrix (dashed) methods compared to the high-frequency approximation (dasheddotted). The driving parameters are $\hbar \Omega=1.15 \mathrm{meV}$ and $V=6.0 \mathrm{mV}$. (b) Corresponding Fano Factor.

\section{COHERENT TRANSPORT SUPPRESSION}

We now turn our attention to the coherent control of current. Tunneling suppression in a closed, driven system is known for more than a decade. For example for a driven bistable potential, tunneling breaks down at exact crossings of the quasi-energy spectrum. Then, one observes the so-called coherent destruction of tunneling [18] which has been studied in a number of cases $[16,19,20]$, but in the context of transport between two leads has received attention only recently $[9,12,21]$.

Surveying the time-averaged current calculated numerically from the transfer-matrix and the tight-binding method plotted in Fig. 2(a), we observe current minima for distinct values of $e V_{\mathrm{ac}} / \hbar \Omega$ for frequencies in the microwave regime. The reason for the current suppressions becomes apparent from the effective tunnel matrix element $\Delta_{\text {eff }}=J_{0}\left(e V_{\text {ac }} / \hbar \Omega\right) \Delta$. This expression implies that the tunneling between the two wells and consequently the current vanishes whenever the ratio $e V_{\mathrm{ac}} / \hbar \Omega$ assumes a zero of the Bessel function $J_{0}$, i.e. for the values $2.405,5.520,8.654, \ldots$ By varying the ratio between driving amplitude and frequency, we can thus tune the tunneling between the two wells and thereby control the current [12]. For a frequency $\Omega=5 \Delta / \hbar$, the analytically obtained current in Fig. 2 shows a remarkable agreement with the tight-binding result.

Figure 2(b) depicts the noise strength of the current for the tight-binding approximation characterized by the Fano factor $F=S / e I$. For zero driving amplitude, we find $F \approx 1 / 2$ which is characteristic for the transport through a double barrier [22]. Note that the central barrier is considerably lower and, thus, the outer barriers determine the transport. At the current suppression, the central barrier becomes the bottleneck. Then, the setup corresponds to a tunneling point contact with $F \approx 1$. In the crossover region, the noise can be even lower than in the static case, i.e. $F<1 / 2$. 


\section{CONCLUSIONS}

We have demonstrated that the current across a double-well heterostructure is strongly affected by the purely coherent influence of an oscillating gate voltage. We have used a transfer-matrix method as an exact approach to compute tunneling currents through such a system. We compared these results to those obtained from a tight-binding Floquet description. In particular, we find that the current suppression is controlled by the ratio of the driving frequency and amplitude. This can be understood by exploring the high-frequency limit within the tight-binding formalism. Since the effective inter-well coupling depends sensitively on the driving parameters, the transport properties of the double well can be controlled. The effective behavior ranging from transport through an almost open channel to a regime of rare tunnel events. The qualitative difference between these transport regimes is also reflected in the behavior of the Fano factor. In the crossover region, the driving can even reduce the noise level.

\section{ACKNOWLEDGMENTS}

We thank S. Camalet, G.-L. Ingold, and J. Lehmann for helpful discussions. This work has been supported through Acción Integrada no. HA2003-0091. Financial support is also acknowledged from MEC (Spain), Grant no. BFM2001-0172, Fundación Ramón Areces, and DFG (Germany), Graduiertenkolleg 283 and SFB 486.

\section{REFERENCES}

1. L. Esaki, and R. Tsu, IBM J. Res. Dev., 14, 61 (1970).

2. T. C. L. G. Sollner, W. D. Goodhue, P. E. Tannenwald, C. D. Parker, and D. D. Peck, Appl. Phys. Lett., 43, 588-590 (1983).

3. M. Grifoni, and P. Hänggi, Phys. Rep., 304, 229 (1998).

4. H. Qin, A. W. Holleitner, K. Eberl, and R. H. Blick, Phys. Rev. B, 64, 241302 (2001).

5. W. G. van der Wiel, S. De Francesoni, J. M. Elzerman, T. Fujisawa, S. Tarucha, and L. P. Kouwenhoven, Rev. Mod. Phys., 75, 1 (2003).

6. G. Platero, and R. Aguado, Phys. Rep., 395, 1 (2004).

7. S. Kohler, J. Lehmann, and P. Hänggi, Phys. Rep., 406, 379 (2005).

8. P. K. Tien, and J. P. Gordon, Phys. Rev., 129, 647 (1963).

9. S. Camalet, S. Kohler, and P. Hänggi, Phys. Rev. B, 70, 155326 (2004).

10. M. Wagner, Phys. Rev. B, 49, 16544 (1994).

11. J. Lehmann, S. Camalet, S. Kohler, and P. Hänggi, Chem. Phys. Lett., 368, 282 (2003).

12. S. Kohler, S. Camalet, M. Strass, J. Lehmann, G.-L. Ingold, and P. Hänggi, Chem. Phys., 296, 243 (2004).

13. S. Camalet, J. Lehmann, S. Kohler, and P. Hänggi, Phys. Rev. Lett., 90, 210602 (2003).

14. R. Landauer, IBM J. Res. Dev., 1, 223 (1957).

15. M. Wagner, and F. Sols, Phys. Rev. Lett., 83, 4377 (1999); Ann. Phys. (Leipzig), 9, 776 (2000).

16. M. Wagner, Phys. Rev. A, 51, 798 (1995).

17. J. H. Shirley, Phys. Rev., 138, B979 (1965).

18. F. Grossmann, T. Dittrich, P. Jung, and P. Hänggi, Phys. Rev. Lett., 67, 516 (1991).

19. M. Holthaus, Phys. Rev. Lett., 69, 351 (1992).

20. C. E. Creffield, and G. Platero, Phys. Rev. B, 65, 113304 (2002).

21. M. Rey, M. Strass, S. Kohler, F. Sols, and P. Hänggi, Chem. Phys. (in press), cond-mat/0412221.

22. Ya. M. Blanter, and M. Büttiker, Phys. Rep., 336, 1 (2000). 